\title{
G51.04+0.07 and its environment: Identification of a new Galactic supernova remnant at low radio frequencies
}

\author{
L. $\operatorname{Supan}^{1,2}$, G. Castelletti ${ }^{1,2}$, W. M. Peters ${ }^{3}$, and N. E. Kassim ${ }^{3}$ \\ ${ }^{1}$ CONICET-Universidad de Buenos Aires, Instituto de Astronomía y Física del Espacio (IAFE), Buenos Aires, Argentina \\ e-mail: lsupan@iafe.uba.ar \\ 2 Universidad de Buenos Aires, Facultad de Ciencias Exactas y Naturales, Buenos Aires, Argentina \\ ${ }^{3}$ Remote Sensing Division, Naval Research Laboratory, Washington, DC 20375, USA
}

Received 9 March 2018 / Accepted 26 April 2018

\begin{abstract}
We have identified a new supernova remnant (SNR), G51.04+0.07, using observations at $74 \mathrm{MHz}$ from the Very Large Array LowFrequency Sky Survey Redux (VLSSr). Earlier, higher frequency radio continuum, recombination line, and infrared data had correctly inferred the presence of nonthermal radio emission within a larger, complex environment including ionised nebulae and active star formation. However, our observations have allowed us to redefine at least one SNR as a relatively small source $(7.5 \times 3$ 'in size) located at the southern periphery of the originally defined SNR candidate G51.21+0.11. The integrated flux density of G51.04+0.07 at $74 \mathrm{MHz}$ is $6.1 \pm 0.8 \mathrm{Jy}$, while its radio continuum spectrum has a slope $\alpha=-0.52 \pm 0.05\left(S_{v} \propto v^{\alpha}\right)$, typical of a shell-type remnant. We also measured spatial variations in the spectral index between 74 and $1400 \mathrm{MHz}$ across the source, ranging from a steeper spectrum $(\alpha=-0.50 \pm 0.04)$ coincident with the brightest emission to a flatter component $(\alpha=-0.30 \pm 0.07)$ in the surrounding fainter region. To probe the interstellar medium into which the redefined SNR is likely evolving, we have analysed the surrounding atomic and molecular gas using the $21 \mathrm{~cm}$ neutral hydrogen $(\mathrm{HI})$ and ${ }^{13} \mathrm{CO}(J=1-0)$ emissions. We found that G51.04+0.07 is confined within an elongated $\mathrm{HI}$ cavity and that its radio emission is consistent with the remains of a stellar explosion that occurred $\sim 6300 \mathrm{yr}$ ago at a distance of $7.7 \pm 2.3 \mathrm{kpc}$. Kinematic data suggest that the newly discovered SNR lies in front of HII regions in the complex, consistent with the lack of a turnover in the low frequency continuum spectrum. The $\mathrm{CO}$ observations revealed molecular material that traces the central and northern parts of G51.04+0.07. The interaction between the cloud and the radio source is not conclusive and motivates further study. The relatively low flux density $(\sim 1.5 \mathrm{Jy}$ at $1400 \mathrm{MHz})$ of G51.04+0.07 is consistent with this and many similar SNRs lying hidden along complex lines of sight towards inner Galactic emission complexes. It would also not be surprising if the larger complex studied here hosted additional SNRs.
\end{abstract}

Key words. ISM: supernova remnants - ISM: individual objects: G51.04+0.07 - radio continuum: general

\section{Introduction}

Supernovae (SNe) explosions and their remnants (SNR) play a major role in key areas across Galactic astrophysics. As the presumed source of Galactic cosmic rays, they seed the interstellar medium (ISM) with at least approximately one third of its energy density and drive its structure and evolution (McKee \& Ostriker 1977), including the stimulation of new generations of star formation. The rate of massive star formation and the Galactic $\mathrm{SNe}$ rate must also be related. These and other factors motivate an accurate census of SNRs as key tracers of both stellar evolution and SNe. Despite considerable effort, the number of identified Galactic SNRs, 300 (Green 2017), remains significantly lower than expected due to severe observational selection effects. Indeed, statistical studies, including OB stars counts, pulsar birth rates and supernova rates in other Local Group galaxies predict that there should be approximately one to two stellar explosions per century in our Galaxy (Li et al. 1991; Tammann et al. 1994). For example, by assuming that the radio synchrotron emission from an SNR is observable for 50000 to $100000 \mathrm{yr}$, the number of Milky Way SNRs should be at least three times greater than currently known. The same conclusion on the Galactic remnant's population had already been reached by Helfand et al. (1989) on the basis of the number and distribution of SNRs in the Galaxy by then catalogued (Green 1988).

The evidence is clear that the "missing" SNRs include both the youngest and oldest SNRs. The former were missed by the early generations of single-dish Galactic plane surveys that identified SNRs based on their extended morphology but at limited angular resolution. The latter, the older extended SNRs, were also missed due to limited dynamic range in surface brightness sensitivity, leaving them confused along complex Galactic sight-lines including the superposition of thermal and nonthermal sources of varying sizes and brightnesses. For several decades now, observations have proceeded painstakingly, uncovering a few new SNRs per year, at best (see for instance, Brogan et al. 2004; Tian et al. 2008; Roy \& Pal 2013; Sabin et al. 2013; Green et al. 2014; Kothes et al. 2017). A significant step forwards filling out the census of Galactic SNRs was achieved with the $330 \mathrm{MHz}$ system on the legacy Very Large Array (VLA; Brogan et al. 2006). Together with a rich archive of higher frequency radio and infrared observations to distinguish them from HII regions, this $330 \mathrm{MHz}$ interferometric survey uncovered 35 new SNRs in a relatively limited region of the inner Galaxy. This work illustrated the advantage of lower frequency interferometer observations in overcoming selection effects that have hidden many SNRs, and suggests a resurgence in higher 
angular resolution, low-frequency observations could impact the field significantly. Newer surveys like GLEAM (Wayth et al. 2015; Hurley-Walker et al. 2017) could be particularly potent as they probe into regions of the inner Galaxy unreachable from northern facilities.

In this paper, we present compelling evidence for a new Galactic SNR, named G51.04+0.07. The unambiguous identification of its nonthermal radio continuum emission was revealed at $74 \mathrm{MHz}$ as part of the Very Large Array Low-frequency Sky Survey Redux (VLSSr, Lane et al. 2014). These data are used in conjunction with available higher-frequency radio images to constrain the continuum spectrum of the source. We have also investigated the properties of the ISM into which the SNR G51.04+0.07 is evolving, by using public datasets from the $21 \mathrm{~cm}$ VLA Galactic Plane Survey (VGPS, Stil et al. 2006) and ${ }^{13} \mathrm{CO}(J=1-0)$ observations from the Boston University-Five College Radio Astronomy Observatory Galactic Ring Survey (GRS, Jackson et al. 2006).

\section{Results}

\subsection{The radio continuum emission: morphology and spectrum}

Recently, Anderson et al. (2017) presented a list of new SNR candidates in the Galaxy identified on the basis of $1-2 \mathrm{GHz}$ continuum data from The $\mathrm{HI}, \mathrm{OH}$, Recombination line survey of the Milky Way (THOR) combined with those from the VLA 1.4 GHz Galactic Plane Survey (VGPS) and infrared survey observations. The large $\left(\sim 30^{\prime}\right)$, complex Galactic region, G51.21+0.11, with an integrated flux density at $1400 \mathrm{MHz}$ of $24.35 \mathrm{Jy}$, is part of their compilation of candidates. Our observations, including new data at $74 \mathrm{MHz}$, have allowed us to take the next step in disentangling the superposition of thermal and nonthermal emission in the complex. As a result, we ascertain that the complex likely contains, not unexpectedly, multiple SNRs and HII regions. In this paper we focus on a region of nonthermal emission that we define as the SNR G51.04+0.07, which is considerably smaller than the original classification of the larger SNR candidate made by Anderson et al. (2017). We note that there is evidence of nonthermal emission elsewhere in the complex, indicating the possible presence of at least one more SNR.

Figure 1 shows a three-colour map of the region corresponding to the source G51.21+0.11. The image compares the infrared Midcourse Space Experiment (MSX) image at $8 \mu \mathrm{m}$ (red; Price et al. 2001) with superimposed total intensity radiocontinuum images from the VLSSr and VGPS at $74 \mathrm{MHz}$ (blue) and $1420 \mathrm{MHz}$ (green), respectively. The image at the highest radio frequency combines data from the $\mathrm{D}$ configuration of the VLA with Effelsberg single dish observations (Stil et al. 2006) ${ }^{1}$. The MSX- $8 \mu \mathrm{m}$ image traces thermal gas emission from polycyclic aromatic hydrocarbons, characteristic of HII regions. At $1420 \mathrm{MHz}$ both thermal and nonthermal emission components are detected. Nevertheless, at such low frequencies as that of the VLSSr nonthermal features dominate the band. Within this context, the information provided by the $74 \mathrm{MHz}$ data from the VLSSr is unique for spatially segregating the thermal and nonthermal emission and constraining the morphology and flux density of the SNR candidate emission. In the field of view shown in Fig. 1, a distinctive cyan feature approximately at the

\footnotetext{
1 Because the area located to the north-east of G51.04+0.07 is not covered by the "THOR + VGPS" data (Beuther et al. 2016), we only used the observations provided by the VGPS to produce the multi-frequency comparison presented in Fig. 1.
}

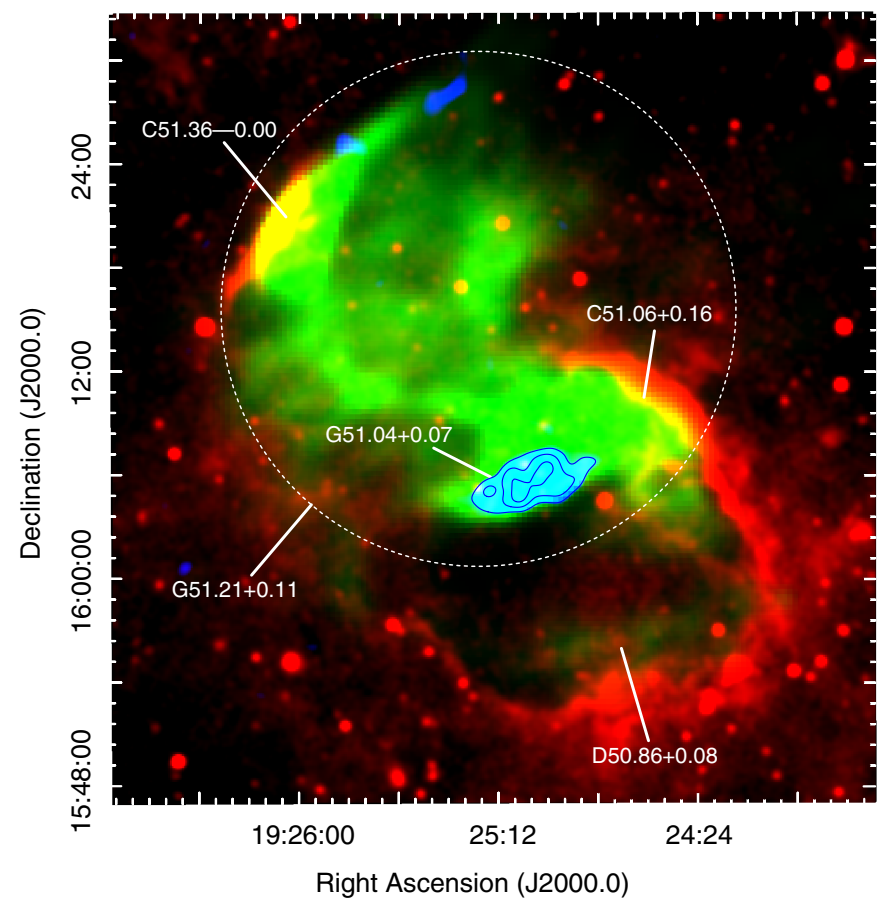

Fig. 1. Colour composite image showing the spatial correspondence between the mid-infrared emission as observed by MSX at $8 \mu \mathrm{m}$ (in red), and the radio continuum emission at 74 (in blue) and $1420 \mathrm{MHz}$ (in green) from the VLSSr and VGPS, respectively. Thermal emission features are traced in yellow and green, while nonthermal radiation is visible in cyan. The overlaid contours tracing the $74 \mathrm{MHz}$ nonthermal emission from the new SNR G51.04+0.07 are at 0.34, 0.58, and $0.78 \mathrm{Jy}_{\text {beam }}{ }^{-1}$. The dotted circle indicates the size and position of the previously proposed SNR candidate G51.21+0.11 (Anderson et al. 2017). The HII regions in the field are also labelled.

center of the field, where both the emission at 74 and $1420 \mathrm{MHz}$ positionally coincide, denotes a region where purely nonthermal emission occurs. Although the VLSSr has limited sensitivity to diffuse structures on $30^{\prime}$ scales, we would still expect to detect smaller emission knots and the s-shaped ridge of emission visible in the VGPS image if that emission were nonthermal. The yellow structures in our colour representation trace thermal regions where the radio emission at $1420 \mathrm{MHz}$ and the mid-infrared radiation overlap. The agreement between these two spectral bands observed to the north-east and west in the field of view corresponds to the photodissociation regions associated with the HII regions C51.36-0.00 (centered at $\left.\mathrm{RA} \sim 19^{\mathrm{h}} 26^{\mathrm{m}} 00^{\mathrm{s}}, \mathrm{Dec} \sim 16^{\circ} 20^{\prime} 00^{\prime \prime}, \mathrm{J} 2000.0\right)$ and C51.06+ 0.16 (centered at $\sim 19^{\mathrm{h}} 24^{\mathrm{m}} 48^{\mathrm{s}}, \sim 16^{\circ} 09^{\prime} 00^{\prime \prime}$ ) in the catalogue presented by Anderson \& Bania (2009). Regarding the HII region D50.86+0.08 $\left(\sim 19^{\mathrm{h}} 24^{\mathrm{m}} 42^{\mathrm{s}}, \sim 15^{\circ} 56^{\prime} 00^{\prime \prime}\right)$, we note that because of the low surface brightness observed at $1420 \mathrm{MHz}$ it does not appear as a bright yellow feature (see Fig. 1). For this and the C51.06+0.16 HII region, Anderson \& Bania (2009) derived kinematical distances of $\sim 8 \mathrm{kpc}$. This was based on recombination-line emission detected at $42.9 \pm 1.2 \mathrm{~km} \mathrm{~s}^{-1}$ and $42.2 \pm 0.5 \mathrm{~km} \mathrm{~s}^{-1}$, respectively, with the NRAO Green Bank 140-foot telescope, together with existing sky surveys of neutral hydrogen $(\mathrm{HI})$ and ${ }^{13} \mathrm{CO}^{2}$. Although no radio recombination line detection towards the HII region C51.36-0.00 has been

\footnotetext{
2 As described by Anderson \& Bania (2009), uncertainties up to $2 \mathrm{kpc}$ in their analysis can result from velocity deviations of $\sim 10 \mathrm{~km} \mathrm{~s}^{-1}$ caused by the streaming motions in the HI gas.
} 
reported in the literature, the catalogued distance to this thermal source is $\sim 6.4 \mathrm{kpc}$ (Anderson \& Bania 2009). We also note that in the surveyed area, two sites of massive star formation (Lumsden et al. 2013) as well as spots of $\mathrm{H}_{2} \mathrm{O}$ and $\mathrm{CH}_{3} \mathrm{OH}$ maser emission (Codella et al. 1994; Szymczak et al. 2012) were identified. Finally, as alluded earlier, marginal evidence for nonthermal emission at the north-east of Fig. 1 may indicate the presence of at least one more SNR in the larger complex.

Even though it was not previously recognised as a distinct zone, an elongated nonthermal structure is now evident in the field. In Fig. 2 we present a zoomed view of this region, underscored by its emission at $74 \mathrm{MHz}$. As mentioned above, we refer to this region as the source G51.04+0.07 (hereafter, G51.04), according to its approximate geometric center. In what follows, we provide morphological and spectral evidence of the nature of this source as a new SNR in our Galaxy. Clearly the $74 \mathrm{MHz}$ emission identifying G51.04 is significantly less extended than G51.21+0.11, the latter being a composite of both thermal and nonthermal emission, although previously classified by Anderson et al. (2017) as a single SNR. This is not necessarily surprising, as a mixture of thermal and nonthermal radio emission in such area was mapped by the THOR + VGPS data.

From the low-frequency radio image, the emission from G51.04 covers an elliptical region with major and minor axes of $7.5 \times 3$ 'in the south-east and north-west direction. The large aspect ratio of the source $(\sim 2.5: 1)$ might be associated with an inhibited expansion in the direction of the Galactic plane. However, no significant brightening is observed in this direction, as one might expect due to enhanced compression. With an angular resolution of $75^{\prime \prime}$ and a sensitivity of $0.095 \mathrm{Jy}_{\text {beam }}^{-1}$, the image at $74 \mathrm{MHz}$ reveals that the brightest emission in G51.04 occurs, at least as we see projected on the plane of the sky, inside the radio shell. A maximum with brightness $0.15 \mathrm{Jy}^{\circ}$ beam $^{-1}$ located at $\sim 19^{\mathrm{h}} 25^{\mathrm{m}} 07^{\mathrm{s}}, \sim 16^{\circ} 05^{\prime}$ is noticeable.

We considered, but disfavour, an interpretation of the bright central feature as a pulsar driven nebula (PWN) for one reason: its continuum spectrum ( $\alpha \sim-0.5$, see below) is too steep, and PWNs normally have relatively flat spectra. Moreover, no radio pulsar coincident with G51.04 has been detected in the latest ATNF Pulsar Catalogue ${ }^{3}$ (Manchester et al. 2005), version 1.58 (accessed 21 June, 2018), with the nearest pulsar, PSR J1926+1613, located $15^{\prime}$ away. Our search also yields negative results in other pulsar surveys ${ }^{4}$. A fainter radio spot $\left(\sim 0.1 \mathrm{Jy} \mathrm{beam}^{-1}\right)$ to the north-west near $\sim 19^{\mathrm{h}} 25^{\mathrm{m}} 02^{\mathrm{s}}, \sim 16^{\circ}$ $06^{\prime}$ is also resolved in the low-frequency image. The brightness gradually decreases in strength towards the edges of the source. The boundaries of G51.04 are not particularly sharp.

The integrated flux density of $\mathrm{G} 51.04$ at $74 \mathrm{MHz}$ is $6.1 \pm 0.8 \mathrm{Jy}$. We computed the error in this estimate by taking several sources of uncertainty into account (added in quadrature), the main of which are the ones associated with intrinsic errors in the calibration of the absolute flux-density scale $(\sim 15 \%)$, clean bias errors modified for extended sources, and uncertainties in defining the outer boundary of the radio emission.

On the basis of the flux density measurement at $74 \mathrm{MHz}$ for G51.04 combined with higher frequency measurements from radio surveys reported in Table 1, we estimated the radio

\footnotetext{
http://www.atnf.csiro.au/research/pulsar/psrcat/

4 See for instance http://www.ioffe.ru/astro1/psr-catalog/ Catalog.php and https://confluence.slac.stanford.edu/ display/GLAMCOG/Public+List+of+LAT-Detected+Gamma-Ray+ Pulsars
}

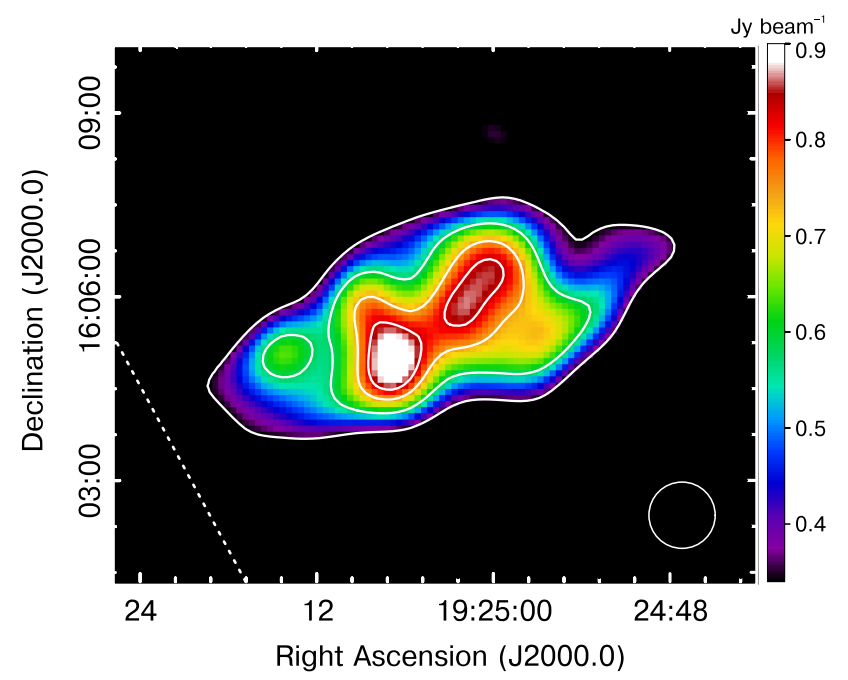

Fig. 2. A close view of the newly identified SNR G51.04 at $74 \mathrm{MHz}$ from the VLSSr. This image is strong evidence for the nonthermal nature of the emission and is currently the only available image of this source below $100 \mathrm{MHz}$. The measured sensitivity level is $0.095 \mathrm{Jy} \mathrm{beam}^{-1}$, and the contours are traced at 0.34, 0.48, 0.78, and $0.83 \mathrm{Jy}$ beam $^{-1}$. The synthesized beam shown at the bottom right corner is $75^{\prime \prime} \times 75^{\prime \prime}$. The position and direction of the Galactic plane are indicated by the dotted line.

continuum spectrum of the source. All the measured flux densities were tied to the flux scale of Perley \& Butler (2017). In Fig. 3 we show a plot of the integrated spectrum for G51.04. A weighted fit to the data points using a single power law yields a slope $\alpha=-0.52 \pm 0.05$ (defined by the relation $S_{v} \propto v^{\alpha}$, with $S_{v}$ the flux at the frequency $v$ ). This result shows conclusively that the radiation from G51.04 has a nonthermal origin. Moreover, the derived spectrum is entirely in concordance with spectral indices measured in many other recognised shell-type Galactic SNRs (for which the mean spectral index is $\alpha \sim-0.5$, Kovalenko et al. 1994). It is noteworthy that in spite of the thermal emission present in the larger G51.21+0.11 Galactic complex, the spectrum of G51.04 does not show evidence of a turnover at frequencies $v<100 \mathrm{MHz}$. Low frequency spectral turnovers towards Galactic SNRs are common but not ubiquitous, and are attributed to an inhomogeneous component of low density ionised gas surrounding normal HII regions along the line of sight (Kassim 1989). Absorption from ionised gas has now been spatially resolved by the VLA at $74 \mathrm{MHz}$, towards, for example, the SNRs W49B (Lacey et al. 2001), 3C 391 (Brogan et al. 2005), and IC 443 (Castelletti et al. 2011). In the two latter cases the absorption indicates the SNRs are clearly interacting with a surrounding molecular cloud complex. The lack of clear absorption towards G51.04 at $74 \mathrm{MHz}$ might indicate that it is in the foreground relative to the larger G51.21+0.11 complex.

Using the integrated flux density estimate and the angular extent measured at $74 \mathrm{MHz}$, the surface brightness of the newly discovered SNR G51.04, $\Sigma=1.5 \times 10^{-19}\left(S / \theta^{2}\right) \mathrm{W} \mathrm{m}^{-2} \mathrm{~Hz}^{-1} \mathrm{sr}^{-1}$, where $S$ and $\theta$, respectively, represent the flux (in Jy) and the angular size (in arcmin) of the source, is $\Sigma_{74 \mathrm{MHz}} \simeq 4.1 \times 10^{-20} \mathrm{~W} \mathrm{~m}^{-2} \mathrm{~Hz}^{-1} \mathrm{sr}^{-1}$. For a reference frequency of $1 \mathrm{GHz}$, we obtained $\Sigma_{1 \mathrm{GHz}} \simeq 1 \times 10^{-20} \mathrm{~W} \mathrm{~m}^{-2} \mathrm{~Hz}^{-1} \mathrm{sr}^{-1}$. This result is consistent with the range presented by Pavlović et al. (2013), encompassing values $10^{-21}-10^{-19} \mathrm{~W} \mathrm{~m}^{-2} \mathrm{~Hz}^{-1} \mathrm{sr}^{-1}$ at $1 \mathrm{GHz}$ for a large sample Galactic SNRs. This surface brightness ranks G51.04 at about thirty out of $\sim 180$ objects classified as shell-like SNRs (Green 2017). This suggests that G51.04 is one 
Table 1. Flux densities used to construct the global radio continuum spectrum of G51.04.

\begin{tabular}{ccl}
\hline \hline $\begin{array}{c}\text { Frequency } \\
(\mathrm{MHz})\end{array}$ & $\begin{array}{c}\text { Flux density } \\
(\mathrm{Jy})\end{array}$ & \multicolumn{1}{c}{ Survey } \\
\hline 74 & $6.1 \pm 0.8$ & VLSSr (Lane et al. 2014) \\
327 & $2.7 \pm 0.8$ & WSRT 327 MHz Galactic Plane Survey (Taylor et al. 1996) \\
1400 & $1.5 \pm 0.3$ & THOR + VGPS (Beuther et al. 2016) \\
1400 & $1.2 \pm 0.2$ & NRAO VLA Sky Survey (Condon et al. 1998) \\
4850 & $0.7 \pm 0.2$ & 4.85 GHz sky survey (Condon et al. 1994) \\
\hline
\end{tabular}

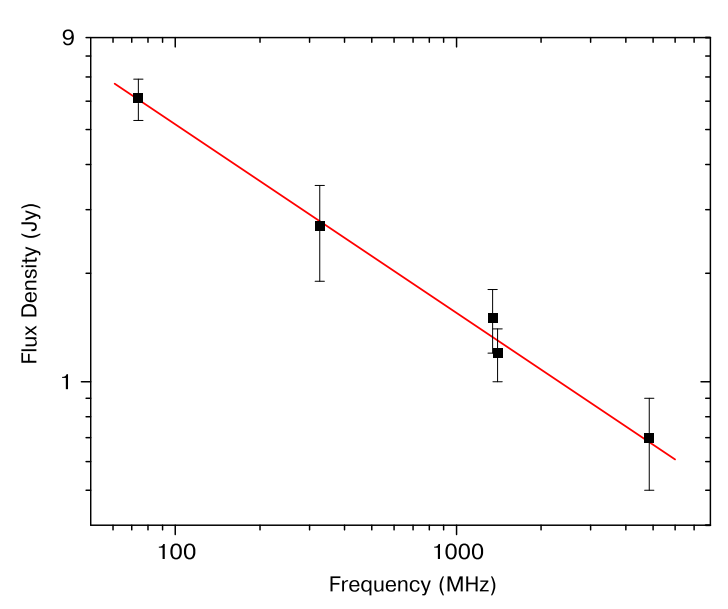

Fig. 3. Global radio continuum spectrum of G51.04 obtained from the flux density measurements summarised in Table 1 . The straight line represents a weighted fit performed to all the plotted values with a powerlaw index $\alpha=-0.52 \pm 0.05$.

of the high surface brightness SNRs known to missing from catalogues due to observational selection effects. By integrating the spectrum from $10^{7}$ to $10^{11} \mathrm{~Hz}$, we derived a radio luminosity of $\sim 2 \times 10^{33} \mathrm{erg} \mathrm{s}^{-1}$ for a distance to the source of $\sim 7.7 \mathrm{kpc}$ (see Sect. 2.3), consistent with typical radio luminosities of Galactic SNRs (e.g. Ilovaisky \& Lequeux 1972; Pavlović et al. 2013). Taken together, the derived radio properties for G51.04 are all consistent with a SNR origin.

In addition to the global radio continuum analysis, we made spatially resolved spectral measurements searching for trends in the spectral index across G51.04. To accomplish this we used the VLSSr image at $74 \mathrm{MHz}$ together with the image at $1400 \mathrm{MHz}$ from THOR + VGPS. We notice that, because the visibility data were not available for the image at the higher frequency, this dataset was not limited in its $u v$ range nor tapered to match the dirty beam at $74 \mathrm{MHz}$. Hence, the spectral index map shown in Fig. 4 was constructed by convolving the highest frequency image to the $75^{\prime \prime}$ synthesized beam of the VLSSr-74 MHz image. Before calculating spectral indices, the images at 74 and $1400 \mathrm{MHz}$ were also aligned, interpolated to identical projections, and clipped at a $3.5 \sigma$ and $3 \sigma$ significance level of their respective sensitivities (at $1400 \mathrm{MHz}$ the rms noise level and the synthesized beam are $\sim 5 \mathrm{mJy}^{\text {beam }}{ }^{-1}$ and $25^{\prime \prime}$, respectively). The errors in our determination of the spectral index from the map are 0.03-0.09 for the brightest and the faintest regions, respectively. In addition to the results presented here, we emphasise that a good quality image at an intermediate frequency between 74 and $1400 \mathrm{MHz}$ is needed to confirm and improve upon the spectral characteristics of G51.04 derived here.

Figure 4 displays the spatial distribution of the spectral index in the redefined SNR obtained from the direct ratio of the

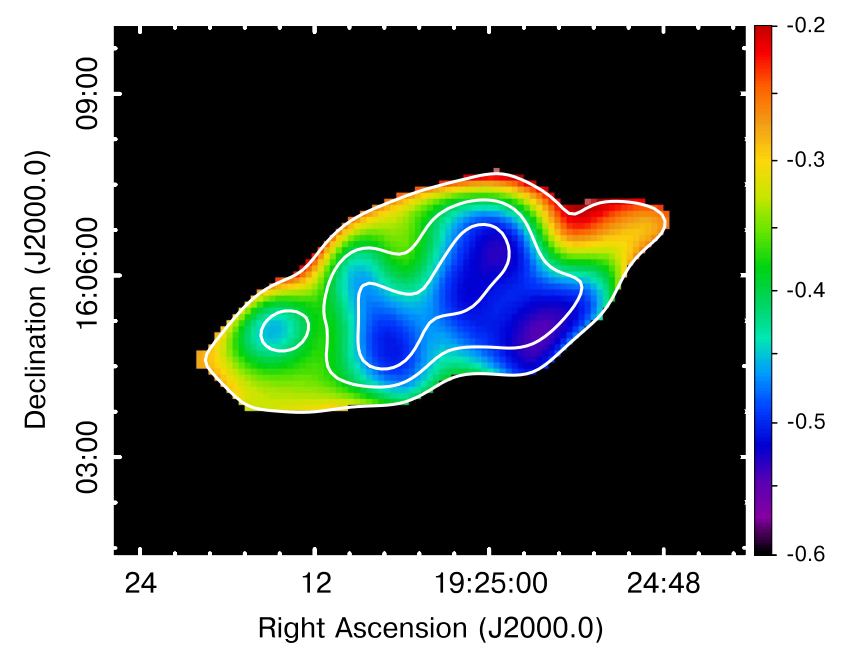

Fig. 4. Spatial spectral index distribution in the SNR G51.04 calculated between 74 and $1400 \mathrm{MHz}$ using the VLSSr and THOR + VGPS images. To create this spectral map, which has a resolution of $75^{\prime \prime}$, the input images at 74 and $1400 \mathrm{MHz}$ were masked at $3.5 \sigma$ and $3 \sigma$ of their respective noise levels. The superimposed contours correspond to the $74 \mathrm{MHz}$ emission at levels $0.34,0.58$, and $0.78 \mathrm{Jy}_{\text {beam }}{ }^{-1}$.

74 and $1400 \mathrm{MHz}$ images. The spread in the spectral indices is of the order of \pm 0.34 across the remnant. A steep spectral index region, with an average value $\alpha_{74}^{1400} \sim-0.5$, is easily identified in this map, which spatially corresponds to the brightest central region in G51.04. The map also reveals a clear trend of a flattening up to $\alpha_{74}^{1400} \sim-0.3$ towards the surrounding lower brightness region. This is most evident in the northwestern border of G51.04. One possibility is that this region is flattened due to the superposition of thermal emitting gas contaminating the remnant's spectral indices, which could be important in the higher frequency image used to construct the spectral map. Overall, we note that the spectral components do not appear to be correlated with features in total intensity, in the sense that the brighter regions are not those with flatter spectrum as is expected under the first-order particle acceleration mechanism. A similar spectral behaviour has been found in the shell-class Galactic SNRs G39.2-0.3, G41.1-0.3 (Anderson et al. 1993), and G15.4+0.1 (Supan et al. 2015), for which it was proposed that different mechanisms are responsible for the spectral and brightness variations observed across these sources.

\subsection{Distance and age determinations}

In the following, we attempt to put constraints on basic parameters for G51.04 such as its age and distance. For the distance estimate we used $21 \mathrm{~cm}$ neutral hydrogen absorption measurements from the VGPS. In Fig. 5 we show the HI emission spectrum 


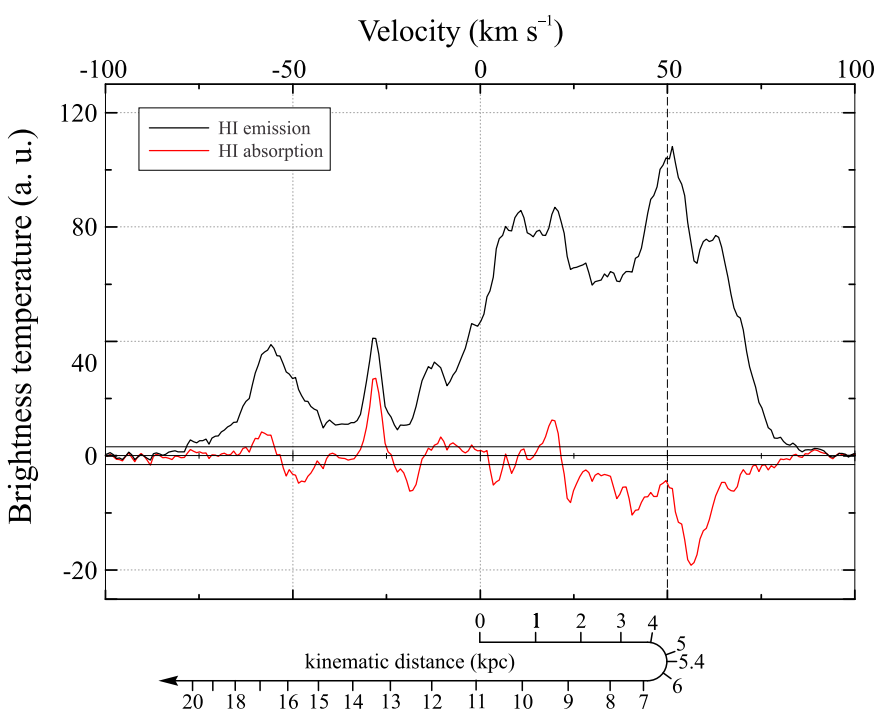

Fig. $5.21 \mathrm{~cm}$ emission (black curve) and absorption (red curve) spectra towards the continuum source G51.04. The dashed vertical line indicates the velocity of the tangent point. The horizontal solid lines correspond to the rms noise level of $3.2 \mathrm{~K}$ in the absorption profile of the HI Below, the assigned kinematic distances are denoted (Fich et al. 1989).

constructed from an area projected against the brightest central portion of the SNR G51.04. The absorption profile is also included in the figure. It was obtained by subtracting the HI emission spectrum from a profile averaged over different patches devoid of continuum emission. Significant and continuous absorption is observed in the velocity range between $20 \mathrm{~km} \mathrm{~s}^{-1}$ and that of the tangential point $\left(v_{\mathrm{TP}} \sim 50 \mathrm{~km} \mathrm{~s}^{-1}\right)$ (all the radial velocities mentioned in this paper are measured with respect to the local standard of rest, LSR). The most positive velocity HI absorption feature at $\sim 56 \mathrm{~km} \mathrm{~s}^{-1}$ likely corresponds to gas at the velocity of the tangential point and could be caused by the expanding motions of the HI gas due to the SNR shockwave. According to the circular rotation model of Fich et al. (1989; with the Galactocentric distance $R_{0}=8.5 \mathrm{kpc}$ and the rotation velocity at the Sun $\Theta=220 \mathrm{~km} \mathrm{~s}^{-1}$ ), the velocity of the tangential point corresponds to a lower limit on the distance of $5.4 \mathrm{kpc}$. At negative velocities a weak absorption feature near $-19 \mathrm{~km} \mathrm{~s}^{-1}$ is observed. However, in view of its association with relative low amplitude emission we regard this feature as unreliable. The same argument holds for the low-level absorption peak at $-50 \mathrm{~km} \mathrm{~s}^{-1}$. In this case, the non-detection of absorption in correspondence with the emission feature in the HI spectrum centered at $-30 \mathrm{~km} \mathrm{~s}^{-1}$ is interpreted as additional support of our suggestion that the feature at the most negative velocity is not genuine. Certainly, if it were real there should be absorption coincident with the emission at $-30 \mathrm{~km} \mathrm{~s}^{-1}$, since there is no kinematic distance ambiguity (or velocity blending) for the gas in this direction of the Galaxy moving at negative velocities. Therefore, in light of the results presented here, an upper limit of $\sim 11 \mathrm{kpc}$ on the kinematic distance to G51.04 can be established, which approximately corresponds to the radial velocity of $0 \mathrm{~km} \mathrm{~s}^{-1}$. We present in Sect. 2.3 a further detailed discussion of this estimate, on the basis of the HI distribution in the direction to G51.04.

For the purpose of deriving the age of G51.04 we note that its angular size suggests it is not an evolved object, a condition that is not particularly constraining. So far, the region has not been observed in $\mathrm{X}$ rays. In examining optical datasets (see for instance, SuperCOSMOS H-alpha Survey, Parker et al. 2005 and The STScI Digitized Sky Survey ${ }^{5}$ ), we did not find obvious correlations with G51.04. To derive an age, we have assumed that the source is in the Sedov-Taylor phase of its evolution. In this evolutionary stage, the age of the remnant is given roughly by $0.4 R_{\mathrm{ST}} / v$, the ratio between the radius and the expansion velocity of the blast wave, being $R_{\mathrm{ST}} \simeq$ $14.5\left(E_{\mathrm{SN}} / 10^{51} \mathrm{erg}\right)^{1 / 5}\left(0.5 \mathrm{~cm}^{-3} / \eta_{0}\right)^{1 / 5} t_{4}^{2 / 5} \mathrm{pc}$, for a shock with a specific heat ratio $\gamma=5 / 3$ (Tielens 2005). Here, $E_{\mathrm{SN}}$ is the energy of the supernova, $\eta_{0}$ is the number density of hydrogen, while $t_{4}$ is the age of the remnant normalised to $10^{4} \mathrm{yr}$. Therefore, using a measured mean density of atomic hydrogen in the ambient $\eta_{0} \sim 10 \mathrm{~cm}^{-3}$ (as we explain later in Sect. 2.3), we calculated an age for the SNR of about $6300 \mathrm{yr}$. It represents an average between the ages corresponding to the major and minor semiaxis of G51.04 (3.75 $\times 1.5$, or 8.4 and $3.4 \mathrm{pc}$ calculated at a distance of $7.7 \pm 2.3 \mathrm{kpc}$, see Sect. 2.3). We emphasise that this is a very rough estimate due to the uncertainties in the measurements involved and the assumption of the Sedov stage in the SNR's evolution.

\section{3. $\mathrm{HI}$ and $\mathrm{CO}$ environments}

To analyse the environs of G51.04, we used observations of the neutral gas emission in the $21 \mathrm{~cm}$ line and ${ }^{13} \mathrm{CO}(J=1-0)$ molecular data from the VGPS and the GRS, respectively. The inspection of the atomic gas throughout the range of velocities covered by the VGPS strikingly shows, in the radial velocity range from $\sim 25$ to $\sim 55 \mathrm{~km} \mathrm{~s}^{-1}$, a distortion in the $\mathrm{HI}$ distribution at the position of the synchrotron emission from G51.04. The distortion resembles a cavity-like structure in the $\mathrm{HI}$ emission, which looks open towards the northern border of G51.04. This feature is presented in Fig. 6, where contours of the radio emission from the VLSSr-74 MHz image are included for reference. To construct this map, an appropriate background level equal to the mean value of each HI image was subtracted. Based on its morphology, we suggest that the HI cavity has possibly been caused by the expansion of the blastwave associated with G51.04 into the HI surroundings. We are aware that similar HI structures have been recognised towards a number of Galactic SNRs, and were interpreted as wind-blown bubbles created by the massive SN progenitor (see for instance, Maxted et al. 2013). However, results obtained from models and observations have also demonstrated that a scenario in which a cavity-like distribution carved by an SNIa progenitor is also plausible (Badenes et al. 2007; Williams et al. 2011). Nevertheless, we highlight here that, based on radio data, we did not find evidence of a PWN inside G51.04.

If we assign a central velocity of $\sim 40 \mathrm{~km} \mathrm{~s}^{-1}$ for the HI cavity, the corresponding near and far kinematic distances are 3.3 and $7.7 \mathrm{kpc}$ (Fich et al. 1989). Based on the hypothesis of association between the cavity-like feature and the source G51.04 (for which we determined from HI absorption measurements a range of $5.4-11 \mathrm{kpc}$ ), we consider the value of $7.7 \pm 2.3 \mathrm{kpc}$ as the most plausible distance for the detected HI structure and the nonthermal radio source G51.04 related to it. The error in this determination includes uncertainties inherent to the circular rotation model and the ones related to the determination of the radial velocity of the HI signature identified in association with G51.04. To complete this picture, we also recall that both the distance and systematic velocities measured for the HII regions in the field of view around G51.04 $\left(v_{\mathrm{RRL}} \sim 42 \mathrm{~km} \mathrm{~s}^{-1}, d \sim 8 \mathrm{kpc}\right.$,

http://archive.stsci.edu/dss/ 


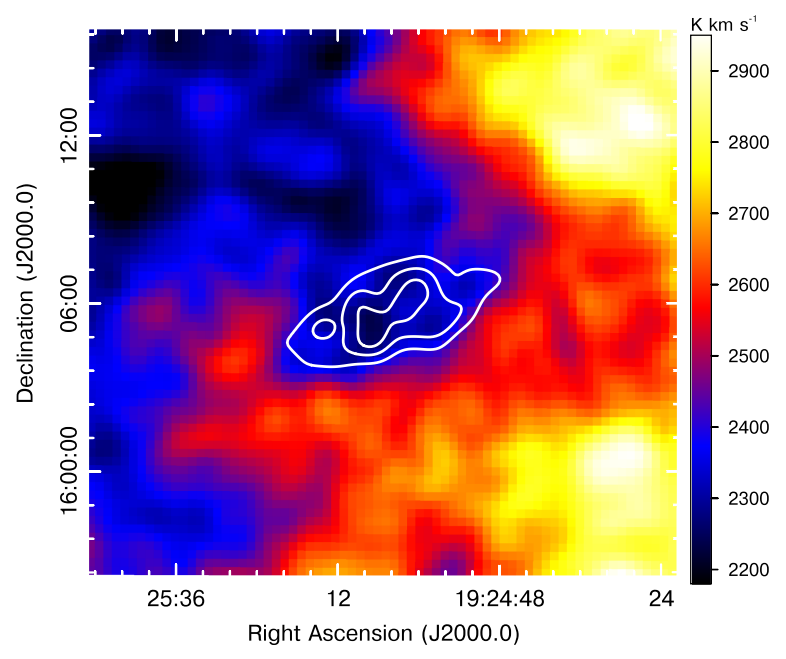

Fig. 6. $21 \mathrm{~cm}$ line emission in the direction of G51.04. The map corresponds to the integration of the neutral gas from 25 to $55 \mathrm{~km} \mathrm{~s}^{-1}$. The $0.34,0.58$, and $0.78 \mathrm{Jy}$ beam $^{-1}$ contours from the $75^{\prime \prime}$ resolution $74 \mathrm{MHz}$ VLSSr image are included to facilitate the comparison between G51.04 and its surroundings.

Lockman et al. (1996); also see our Fig. 1) are similar to the distance determined here for both the HI cavity and the SNR G51.04. Therefore, we conclude that all the observed features, thermal and nonthermal, and the HI are related. Furthermore, taking the lack of a low frequency continuum spectral turnover into account (as discussed in Sect. 2.1), the SNR G51.04 is closer than the HII regions in the complex, or just on their near side.

In determining the physical parameters of the neutral gas that we assume is associated with G51.04, we adopted for the HI cavity a center $\sim 19^{\mathrm{h}} 25^{\mathrm{m}} 05^{\mathrm{s}}, \sim 16^{\circ} 05^{\prime} 44^{\prime \prime}$, a radius of $6^{\prime} \times 3,5$ and a thickness of 2.5 (equal to $\sim 13.4 \times 7.8 \mathrm{pc}$ and $5.6 \mathrm{pc}$ at a distance of $7.7 \mathrm{kpc}$ ). Under the assumption that the $\mathrm{HI}$ emission is optically thin, the HI column density is given as $N_{\mathrm{H}}=1.823 \times 10^{18} \int T_{\mathrm{b}} \mathrm{d} v$, where $T_{\mathrm{b}}[\mathrm{K}]$ and $v\left[\mathrm{~km} \mathrm{~s}^{-1}\right]$ denote the intensity and the velocity of the neutral gas, respectively (Dickey \& Lockman 1990). We thus calculated the average column density for the cavity-like structure to be $N_{\mathrm{H}}=0.74 \times 10^{21} \mathrm{~cm}^{-2}$. We note that this value could be interpreted as an upper limit due to possible confusion with unrelated foreground and background gas. We also found that, at a distance of $7.7 \mathrm{kpc}$, the obtained column density implies an average density in the HI cavity of about $\eta \sim N_{\mathrm{H}} / L=23 \mathrm{~cm}^{-3}$, using a characteristic length of the cavity equal to the mean radius of the HI feature, 4.75 (or $10.6 \mathrm{pc}$ at $d \sim 7.7 \mathrm{kpc}$ ). Therefore, if the neutral gas was originally distributed inside a cylinder of radius and line-of-sight depth of $10.6 \mathrm{pc}$, the derived mean density of hydrogen atoms in the medium is $\eta_{0} \sim 10 \mathrm{~cm}^{-3}$. This estimate is fairly consistent with the $20-50 \mathrm{~cm}^{-3}$ hydrogen density in cold HI clouds (e.g. Dickey \& Lockman 1998; Ferrière 2001). The mean relative error in the determination of parameters for the neutral gas is about $40 \%$, stemming mainly from uncertainties in the distance determination, in the selection of integration boundaries, as well as the inclusion of possible unrelated gas located along the line of sight.

We have also searched for molecular structures traced by the ${ }^{13} \mathrm{CO}(J=1-0)$ gas related to G51.04. After inspecting the entire data cube from the GRS in the velocity range between -5 and $135 \mathrm{~km} \mathrm{~s}^{-1}$, we identified a molecular cloud which seems to match (in projection) the radio emission from G51.04. The integrated emission from this cloud in the LSR range $\sim 40$ $52 \mathrm{~km} \mathrm{~s}^{-1}$ is depicted in Fig. 7a, where contours of the $74 \mathrm{MHz}$ radio emission from VLSSr image are included for ease of comparison. The question to be addressed now is whether this molecular material is part of the complex environment associated with G51.04 and the HII regions. To analyse this point we used the $21 \mathrm{~cm}$ continuum absorption method presented by Roman-Duval et al. (2009) as a tool to determine the distance to the molecular gas. The ${ }^{13} \mathrm{CO}$ average spectrum for the cloud is shown in Fig. 7b. It was extracted from a $\sim 1.5$ box centered at the brightest part of the cloud, that, seen in projection, overlaps the continuum emission from G51.04. Figure 7b also includes the HI absorption profile towards the cloud. It was obtained by subtracting the $\mathrm{HI}$ emission profile from a spectrum averaged over several regions free from ${ }^{13} \mathrm{CO}$ emission. At least two peaks are evident in the ${ }^{13} \mathrm{CO}$ emission line spectrum, one at $\sim 37 \mathrm{~km} \mathrm{~s}^{-1}$ and the other at $\sim 49 \mathrm{~km} \mathrm{~s}^{-1}$. The latter, very close to the velocity of the tangent point, corresponds to the molecular material detected in the direction of G51.04. A correlation between these $\mathrm{CO}$ peaks and $\mathrm{HI}$ absorption features is also noticeable. Because the HI gas is ubiquitously distributed in the Galaxy, at the smaller radial velocity this correlation would be produced by foreground molecular material absorbing the continuum radiation from G51.04. Regarding the $49 \mathrm{~km} \mathrm{~s}^{-1}$ emission line peak, we are aware that if the cloud containing the radio continuum source is at the far kinematic distance $(\sim 6 \mathrm{kpc})$ then the correspondence of $\mathrm{HI}$ absorption features with $\mathrm{CO}$ emission peaks should be observed at velocities greater than the velocity of the cloud of interest (up to the velocity of the tangent point). However, this picture becomes confusing in the case of the discovered cloud. Firstly, although the $49 \mathrm{~km} \mathrm{~s}^{-1}$ emission line peak appears to be broadened up to the velocity of the tangent point, as shown in Fig. 7b, it is difficult to determine whether this feature represents real perturbations of the molecular gas or simply a multiplicity of velocity components along the line of sight. Secondly, CO emission features and the corresponding absorption in the $21 \mathrm{~cm}$ continuum are observed in Fig. $7 \mathrm{~b}$ at a velocity greater than the velocity of the tangent point, but this probably originates in random motions of the gas, that locally can produce velocities greater than those corresponding to the tangent point (Roman-Duval et al. 2009). Therefore, taking all the spectral signatures together, we established a lower limit to the kinematic distance to the cloud at $49 \mathrm{~km} \mathrm{~s}^{-1}$ of $\sim 6 \mathrm{kpc}$, the far distance assigned to this radial velocity (Fich et al. 1989).

We now use our analysis of the molecular gas distribution in conjunction with both the morphological and spectral features observed across G51.04. According to our interpretation of the spectra presented in Fig. 7b, a physical association between the cloud and the radio source G51.04 should not be dismissed (within the uncertainties in the determination of the distances to G51.04 and the cloud). Consequently, as a possibility, the brightest part in the $74 \mathrm{MHz}$ radio emission of G51.04, that we see projected on the plane of the sky lying at the center of the shell, may be in fact indicating a region where the SN shock front is just encountering part of the molecular gas. This does not necessarily mean a critical interaction of G51.04 with its surroundings. The local synchrotron spectrum of G51.04 in this region $(\alpha \sim-0.5)$ is compatible with a first-order Fermi mechanism operating at the shock. It is also interesting to notice that there is $\mathrm{CO}$ molecular gas in apparent coincidence with the localised spectral index flattening observed in the northwestern edge of G51.04. Such a correspondence could be due to a shock that is being decelerated while it is running through the high density 

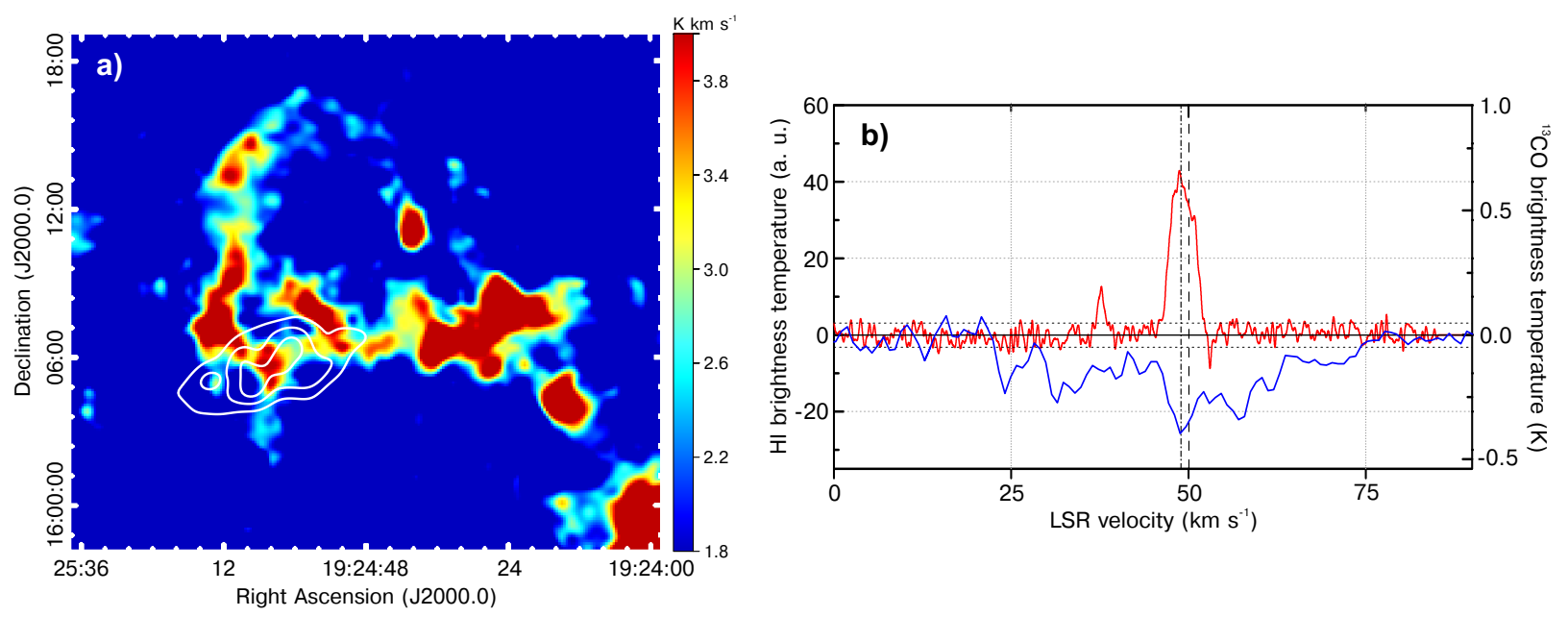

Fig. 7. Panel a: emission of ${ }^{13} \mathrm{CO}(J=1-0)$ in the Galactic complex associated with the source G51.04 and the star forming activity regions shown in Fig. 1. The image was obtained by integrating the molecular line emission in the velocity range from 40 to $52 \mathrm{~km} \mathrm{~s}^{-1}$. The contours trace the $74 \mathrm{MHz}$ radio continuum emission from G51.04 at $0.34,0.58$, and $0.78 \mathrm{Jy}_{\text {beam }}{ }^{-1}$. Panel $b:{ }^{13} \mathrm{CO}$ emission (red curve) and $\mathrm{HI}$ absorption (blue curve) spectra towards the identified molecular component, which are roughly coincident in projection with the radio emission from G51.04. Horizontal dotted lines mark the rms noise of the HI absorption spectrum. The dash-triple-dot line indicates the velocity of the cloud, while the dashed line marks the velocity of the tangent point. The spectral features allow us to establish a lower kinematic distance limit of $\sim 6 \mathrm{kpc}$ to the cloud.

interstellar material. Although this component has a spectrum appreciably flatter than the surrounding synchrotron plasma, no spectral index signature suggesting the presence of thermal absorption from collisional ionisation was found in the postshock region (Brogan et al. 2005; Castelletti et al. 2011). Of course, we recognise that an alternative scenario in which thermal gas associated with the neighbouring HII regions determines the flattening cannot be ruled out. More sensitive radio continuum data is needed before advancing a detailed interpretation of the local spectral variations and its relationship with the observed CO features.

Finally, we point out that an excess of $\gamma$-ray emission at $\mathrm{GeV}$ energies (catalogued as FL8Y J1925.4+1616 ${ }^{6}$ ) has been detected with the Large Area Telescope onboard the Fermi satellite in the G51.21+0.11 complex. To date, the nature of the GeV emission has not been investigated. On the basis of our findings a physical relationship between the Fermi source and the radio emitting components in the field cannot be ruled out. It could represent a new case of a Galactic $\gamma$-ray emitting source related to a complex including the remains of a stellar explosion and star-forming activity in the HII regions.

\section{Concluding remarks}

Low radio frequency observations from the VLSSr at $74 \mathrm{MHz}$ have allowed us to distinguish the nonthermal emission component in a Galactic complex encompassing considerable thermal emission. While earlier work characterised the entire $\sim 30^{\prime}$ complex as the SNR candidate G51.21+0.11, we have been able to isolate the main region of nonthermal emission to less than $10 \%$ of the complex. This allows us to reclassify the SNR candidate as G51.04+0.07 (G51.04). The computed flux density integrated over the whole extent of the emission at $74 \mathrm{MHz}$ is $6.1 \pm 0.8 \mathrm{Jy}$. The unambiguously nonthermal global spectral

\footnotetext{
6 The preliminary Fermi 8-year Point Source List (FL8Y), which will be soon superseeded by the forthcoming Fermi Fourth Source Catalog (4FGL), is available at https://fermi.gsfc.nasa.gov/ssc/data/ access/lat/fl8y/.
}

index $(\alpha \sim-0.52)$ favours an interpretation in which the detected radiation is produced by a SNR probably belonging to the shell-type morphology class. The integrated synchrotron flux density is $\sim 1.51 \mathrm{Jy}$ at the nominal frequency of $1 \mathrm{GHz}$, making G51.04 among the $\sim 15 \%$ of Galactic SNRs whose $1 \mathrm{GHz}$ integrated flux densities are below $2 \mathrm{Jy}$. This is unsurprising since observational selection effects undoubtedly hide many weakly emitting SNRs within numerous inner Galactic complexes. In fact, we cannot rule out that future observations will reveal that the larger complex G51.21+0.11 may harbour additional SNRs. In addition, the lack of a turnover in the integrated spectrum of G51.04 below $100 \mathrm{MHz}$ indicates that the newly defined SNR is closer than the HII regions located in the associated complex at about $8 \mathrm{kpc}$ from us, and possibly immediately on their near side. Our discovery highlights the crucial importance of low radio frequency observations with sufficient angular resolution and surface brightness sensitivity in addressing the "missing SNR problem" in our Galaxy. Addressing this challenge is key to understanding the star formation history of the Galaxy and bears on central problems including the origin of Galactic cosmic rays and the structure and evolution of the interstellar medium.

We have also discovered an elongated HI cavity-like feature whose location and morphology indicate interaction with G51.04. The physical parameters of this structure were used to constrain the kinematic distance and age of the radio continuum source to be equal to $\sim 7.7 \mathrm{kpc}$ and $\sim 6300 \mathrm{yr}$, respectively. Additionally, we identified molecular material that is matching (in projection) the central portion of the SNR, where an enhancement in the radio continuum emission is observed. Despite the suggested morphological implications, the astrophysical connection of the molecular gas emission with the SNR is not straightforward. The correspondence between radio spectral index distribution with the molecular and nonthermal emission at $74 \mathrm{MHz}$ for the brightest central portion of G51.04, suggests the SNR shockwave is encountering molecular material, though not necessarily implying a strong interaction. The situation might be different along the ridge of G51.04, where the flat spectrum 
component suggests the SNR shock is being decelerated during its passage across the dense interstellar material. We did not find convincing spectral evidence of ionisation processes delineated by $74 \mathrm{MHz}$ free-free absorption in this part of the SNR. However such signatures are subtle and higher resolution, low-frequency observations are probably required to detect them, if present.

Acknowledgements. The authors wish to thank the referee Dr. David J. Helfand for his insightful comments and detailed corrections to the manuscript. G. Castelletti is member of the Carrera del Investigador Cientifico of CONICET, Argentina. L. Supan is a post-doc Fellow of CONICET, Argentina. This research was partially supported by grants awarded by ANPCYT (PICT 1759/15) and the University of Buenos Aires (UBACyT 20020150100098BA), Argentina. Basic Research at the Naval Research Laboratory is funded by 6.1 base programs. This publication makes use of molecular line data from the Boston UniversityFCRAO Galactic Ring Survey (GRS) and of data products from the Midcourse Space Experiment.

\section{References}

Acero, F., Ackermann, M., Ajello, M., et al. 2015, ApJS, 218, 23 Anderson, L. D., \& Bania, T. M. 2009, ApJ, 690, 706 Anderson, L. D., Wang, Y., Bihr, S., et al. 2017, A\&A, 605, A58 Anderson, M. C., \& Rudnick, L. 1993, ApJ, 408, 514

Badenes, C., Hughes, J. P., Bravo, E., \& Langer, N. 2007, ApJ, 662,472

Beuther, H., Bihr, S., Rugel, M., et al. 2016, A\&A, 595, A32

Brogan, C. L., Devine, K. E., Lazio, T. J., et al. 2004, AJ, 127, 355

Brogan, C. L., Lazio, T. J., Kassim, N. E., \& Dyer, K. K. 2005, AJ, 130, 148

Brogan, C. L., Gelfand, J. D., Gaensler, B. M., Kassim, N. E., \& Lazio, T. J. W. 2006, ApJ, 639, L25

Castelletti, G., Dubner, G., Clarke, T., \& Kassim, N. E. 2011, A\&A, 534, A21

Codella, C., Felli, M., Natale, V., Palagi, F., \& Palla, F. 1994, A\&A, 291, 261

Condon, J. J., Broderick, J. J., Seielstad, G. A., Douglas, K., \& Gregory, P. C. 1994, AJ, 107, 1829

Condon, J. J., Cotton, W. D., Greisen, E. W., et al. 1998, AJ, 115, 1693

Dickey, J. M., \& Lockman, F. J. 1990, ARA\&A, 28, 215

Draine, B. T., \& Lazarian, A. 1998, ApJ, 494, L19

Ferrière, K. M. 2001, Rev. Mod. Phys., 73, 1031

Fich, M., Blitz, L., \& Stark, A. A. 1989, ApJ, 342, 272
Green, D. A. 1988, Ap\&SS, 148, 3

Green, D. A. 2017, VizieR Online Data Catalog: VII/278

Green, A. J., Reeves, S. N., \& Murphy, T. 2014, PASA, 31, e042

Helfand, D. J., Velusamy, T., Becker, R. H., \& Lockman, F. J. 1989, ApJ, 341,151

Hurley-Walker, N., Callingham, J. R., Hancock, P. J., et al. 2017, MNRAS, 464, 1146

Ilovaisky, S. A., \& Lequeux, J. 1972, A\&A, 18, 169

Jackson, J. M., Rathborne, J. M., Shah, R. Y., et al. 2006, ApJS, 163, 145

Kassim, N. E. 1989, ApJ, 347, 915

Kothes, R., Reich, P., Foster, T. J., \& Reich, W. 2017, A\&A, 597, A116

Kovalenko, A. V., Pynzar', A. V., \& Udal'Tsov, V. A. 1994, Astron. Rep., 38, 78

Lacey, C. K., Lazio, T. J. W., Kassim, N. E., et al. 2001, ApJ, 559, 954

Lane, W. M., Cotton, W. D., van Velzen, S., et al. 2014, MNRAS, 440, 327

Li, Z., Wheeler, J. C., Bash, F. N., \& Jefferys, W. H. 1991, ApJ, 378, 93

Lockman, F. J., Pisano, D. J., \& Howard, G. J. 1996, ApJ, 472, 173

Lumsden, S. L., Hoare, M. G., Urquhart, J. S., et al. 2013, ApJS, 208, 11

Manchester, R. N., Hobbs, G. B., Teoh, A., \& Hobbs, M. 2005, AJ, 129, 1993

Maxted, N. I., Rowell, G. P., Dawson, B. R., et al. 2013, PASA, 30, e055

McKee, C. F., \& Ostriker, J. P. 1977, ApJ, 218, 148

Parker, Q. A., Phillipps, S., Pierce, M. J., et al. 2005, MNRAS, 362, 689

Pavlović, M. Z., Urošević, D., Vukotić, B., Arbutina, B., \& Göker, Ü. D. 2013, ApJS, 204, 4

Perley, R. A., \& Butler, B. J. 2017, ApJS, 230, 7

Price, S. D., Egan, M. P., Carey, S. J., Mizuno, D. R., \& Kuchar, T. A. 2001, AJ, 121,2819

Rengelink, R. B., Tang, Y., de Bruyn, A. G., et al. 1997, A\&AS, 124, 259

Roman-Duval, J., Jackson, J. M., Heyer, M., et al. 2009, ApJ, 699, 1153

Roy, S., \& Pal, S. 2013, ApJ, 774, 150

Sabin, L., Parker, Q. A., Contreras, M. E., et al. 2013, MNRAS, 431, 279

Stil, J. M., Taylor, A. R., Dickey, J. M., et al. 2006, AJ, 132, 1158

Supan, L., Castelletti, G., Joshi, B. C., Surnis, M. P., \& Supanitsky, D. 2015, A\&A, 576, A81

Szymczak, M., Wolak, P., Bartkiewicz, A., \& Borkowski, K. M. 2012, Astron. Nachr., 333, 634

Tammann, G. A., Loeffler, W., \& Schroeder, A. 1994, ApJS, 92, 487

Taylor, A. R., Goss, W. M., Coleman, P. H., van Leeuwen, J., \& Wallace, B. J. 1996, ApJS, 107, 239

Tian, W. W., Leahy, D. A., Haverkorn, M., \& Jiang, B. 2008, ApJ, 679, L85

Tielens, A. G. G. M. 2005, The Physics and Chemistry of the Interstellar Medium, (Cambridge University Press)

Wayth, R. B., Lenc, E., Bell, M. E., et al. 2015, PASA, 32, e025

Williams, B. J., Blair, W. P., Blondin, J. M., et al. 2011, ApJ, 741, 96 